\title{
EMBARAZO POSTERIOR A CIRUGÍA BARIÁTRICA: COMPLICACIONES MATERNAS Y FETALES
}

\author{
Maternal and foetal complications in pregnancy \\ following bariatric (weight-loss) surgery \\ Jairo Hernández-Pinzón*, Marcos Castillo-Zamora, M.D.**, \\ Victoria E. Arango-Galvis, M.D.*** \\ Recibido: marzo 26/08 - Aceptado: septiembre 1/08
}

\section{RESUMEN}

Objetivo: utilizando herramientas de revisiones sistemáticas, describir los efectos de la obesidad en el embarazo para la madre y el feto, así como describir las complicaciones perinatales, intraparto y posnatales en pacientes a las que se les ha practicado cirugía bariátrica.

Metodología: se utilizó la base de datos PubMed/ Medline, los términos de búsqueda fueron: bariatric surgery AND/OR obstetric complications, pregnance, abortion, pregnancy outcomes, adverse pregnancy outcomes. Se hizo énfasis en guías de práctica clínica, se realizó búsqueda en sitios de sinopsis de la literatura médica del Database of Abstracts of Reviews of Effectiveness (DARE).

Resultados: se seleccionaron 50 artículos, los cuales fueron sintetizados en la sección de resultados de la revisión. La cirugía bariátrica predispone a la isquemia intestinal y formación de hernias en los embarazos posteriores. También se han descrito deficiencias específicas de micronutrientes. Por otra parte, existen informes contradictorios sobre el efecto en el peso fetal.

* Médico Interno Clínica Universitaria Teletón. Integrante Grupo de Investigación: Medicina Familiar y Salud de la Población, Universidad de la Sabana. Bogotá, Colombia. Correo electrónico: jairo.hernandez@ unisabana.edu.co

** Especialista en Ginecólogía y Obstetricia. Profesor auxiliar, Universidad de la Sabana. Bogotá, Colombia.

*** Especialista en Cirugía General. Profesora adscrita, Universidad de la Sabana. Bogotá, Colombia.
Conclusiones: el seguimiento de los embarazos después del primer año de la cirugía para pérdida de peso no informa episodios importantes de malnutrición materna o fetal.

La evidencia muestra que los resultados del embarazo después de la cirugía bariátrica son favorables, en comparación con los resultados en los obesos no tratados quirúrgicamente, se necesitan más estudios prospectivos diseñados cuidadosamente para evaluar áreas de incertidumbre en este campo.

Palabras clave: cirugía bariátrica, complicaciones obstétricas, embarazo, obesidad.

\section{SUMMARY}

Objective: using systematic review tools for describing the effects of obesity on pregnancy for the mother and the foetus, as well as describing perinatal, intrapartum and postnatal complications in patients who have undergone bariatric (weigh-loss) surgery.

Methodology: the PUBMED/MEDLINE database was searched using the following search terms: bariatric surgery AND/OR obstetric complications, pregnancy, abortion, pregnancy outcome, adverse pregnancy outcome), emphasizing clinical practice guidelines. Sites giving synopses of the medical literature from the Database of Abstracts of Reviews of Effectiveness (DARE) were searched. 
Results: 50 articles were selected which were synthesised in the review's results section. Bariatric surgery predisposes itself to intestinal ischemia and the formation of hernias in later pregnancies. Specific micronutrient deficiencies have also been described. Some contradictory reports deal with the effect on foetal weight.

Conclusions: no reports regarding important episodes of maternal or foetal malnutrition were found when following-up pregnancies after the first year following surgery for weight-loss.

The evidence revealed that the results of pregnancy following bariatric surgery were favourable compared to results in obese women who had not been surgically treated. Further carefully-designed prospective studies are needed for evaluating areas of uncertainty in this field.

Key words: bariatric (weight-loss) surgery, obstetric labor complication, pregnancy, obesity.

\section{INTRODUCCIÓN}

La obesidad se ha convertido en una condición epidémica en el mundo entero. ${ }^{1-4}$ En Colombia para el año 2005, la encuesta nacional de demografía y salud informó que alrededor del $40 \%$ de la población femenina entre 15 y 49 años presentaba sobrepeso; ${ }^{5}$ de este porcentaje, el 14\% tenia obesidad mórbida. ${ }^{6}$

Hoy en día se plantea la cirugía bariátrica como una posible solución a los problemas de obesidad, considerando que estos procedimientos proveen el único método confiable para perder y sostener la pérdida de peso en pacientes con obesidad mórbida. ${ }^{7,8}$

En la década pasada, la cirugía bariátrica se expandió rápidamente para controlar la epidemia de la obesidad mórbida, y se espera un crecimiento exponencial en los próximos años. ${ }^{9}$ De las personas que consultan para este tipo de procedimientos, $75-85 \%$ son mujeres, cuya edad media es aproximadamente 40 años, lo que refleja un porcentaje importante de mujeres en plena edad reprodroductiva, como se reportó en Estados Unidos en el año 2004, donde la mitad de las 100.000 cirugías bariátricas fueron realizadas en mujeres entre los 18 y 40 años. ${ }^{10,11}$
El embarazo de este grupo de pacientes nos enfrenta a una nueva serie de preguntas y preocupaciones sobre ellas y sus hijos, presentando a los ginecólogos generales y subespecialistas nuevas interrogantes como: ¿cuál es la dieta que se debe aconsejar en estos pacientes?, ¿se altera la absorción de micronutrientes y vitaminas para el desarrollo fetal?, ¿hay algún tipo de efecto sobre el peso fetal?, ¿hay complicaciones perinatales y posnatales en el recién nacido?, ¿existe alguna complicación intraparto en la paciente a la que se le ha practicado cirugía bariátrica?

Los informes de los resultados del embarazo en mujeres que han sido sometidas previamente a cirugía bariátrica son muy escasos, limitados y contradictorios; nuestro objetivo con la presente revisión es, mediante herramientas de revisiones sistemáticas, describir las complicaciones perinatales, intraparto y posnatales en pacientes a las que se les ha practicado cirugía bariátrica.

\section{METODOLOGÍA}

Se realizó una búsqueda en la base de datos PubMed/ Medline (http://www.pubmed.com) con los términos de MeSH database (obesity and pregnancy and bariatric surgery, obstetric complications or abortion or adverse pregnancy outcomes). También se hizo énfasis en la búsqueda de guías de práctica clínica en los siguientes sitios: Guideline Clearinghouse (http://www.guideline.gov/), en el Guidelines Finder de la National Electronic Library for Health del NHS británico (http://www.library. nhs.uk/guidelinesfinder/), en CMA Infobase (http:// mdm.ca/cpgsnew/cpgs/index.asp) y en el Database of Abstract or Review o Effectiveness (DARE).

\section{RESULTADOS}

Se seleccionaron 50 artículos que presentan la siguiente información:

La obesidad, en particular la abdominal, se asocia con riesgos aumentados para diferentes enfermedades. ${ }^{12}$ En el embarazo la obesidad incrementa el riesgo materno (trabajo de parto pretérmino, cesárea primaria o parto instrumentado, 
morbilidad perioperatoria, hipertensión inducida por el embarazo, diabetes gestacional, ganancia de peso excesiva durante todas las fases del embarazo, ${ }^{13,14}$ infección puerperal, disminución de las tasas de iniciación o continuación de la lactancia materna, ${ }^{15}$ riesgos con la anestesia perioperatoria). ${ }^{16}$ También están descritos unos riesgos propios para el feto, como mayor probabilidad de muerte intrauterina, anormalidades de crecimiento fetales, macrosomía, retardo del crecimiento intrauterino, ${ }^{17}$ anomalía congénita ${ }^{15}$ y neonatal (mayor probabilidad de muerte neonatal). Es por esto que la American College of Obstetricians and Gynecologists recomienda a los obstetras proporcionar un adecuado asesoramiento preconcepcional y educación acerca de las posibles complicaciones obstétricas que pueden tener las pacientes obesas e insistir en llevar a cabo un programa de reducción de peso antes de intentar el embarazo. ${ }^{16}$

Se requiere un adecuado aumento de peso materno para permitir un crecimiento fetal normal, un desarrollo y una programación metabólica adecuada. El incremento excesivo de peso puede conducir a aumento del riesgo obstétrico, como macrosomía y retención del peso posparto. El (NIH) en 1990 publicó las recomendaciones de aumento total de peso durante la gestación, basados en el IMC. ${ }^{18} \mathrm{Di}$ ferentes estudios clínicos reportan que la pérdida de peso antes del embarazo mejora la fertilidad y disminuye las complicaciones obstétricas. ${ }^{19-23}$

Respecto a la cirugía bariátrica como opción terapéutica preconcepcional al problema de la obesidad en el embarazo, sus efectos sobre la futura madre y las complicaciones maternas o fetales, se encontró la siguiente información:

Los procedimientos quirúrgicos bariátricos reducen la ingesta calórica, modificando la anatomía gastrointestinal. Estas operaciones son clasificadas como restrictivas y malabsortivas. ${ }^{11}$

Los diferentes tipos de cirugía bariátrica tienen una serie de complicaciones postoperatorias comunes para todas ellas, la de más alta prevalencia en este tipo de pacientes es el "Dumping Síndrome", incluye síntomas como rubor facial, lipotimias, palpitaciones, fatiga y diarrea; ocurre en no menos del 70\% de los pacientes, después que se han sometido a una cirugía bariátrica de tipo malabsortivo, ${ }^{24}$ este síndrome es provocado por la ingestión de azúcar concentrada. ${ }^{25,26}$

Se informan con frecuencia las carencias de hierro, calcio, folato, vitamina B12 y otras sustancias nutritivas; este tipo de deficiencias nutricionales se presentan más comúnmente después de procedimientos malabsortivos (bypass), entre más distal se realice la anastomosis, la desnutrición de proteínas y las carencias de las vitaminas liposolubles (A, D, E, y K) pueden ocurrir con una mayor frecuencia. ${ }^{19,20}$ Otras complicaciones gastrointestinales incluyen obstrucción intestinal, fístulas, estenosis, erosiones, úlceras, adherencias, hernias internas e incisionales y colelitiasis. ${ }^{21,22}$

Por otro lado, algunos pacientes suelen requerir una reintervención subsecuente; un estudio multicéntrico de cohortes ${ }^{27}$ reportó una tasa de reoperación del 3,7\%, y una de readmisión en 30 días del 6,4\%. En otro estudio retrospectivo de serie de casos, analizaron el bypass vs. Y de Roux. El 19,3\% y el 40,4\% fueron hospitalizados de nuevo dentro de 12 a 36 meses después del procedimiento, comparados con los que habían sido admitidos para el cuidado hospitalario 12 ó 16 meses antes del procedimiento (el 7,9\% y el 19,4\%, respectivamente). ${ }^{28}$

También se reportan casos como isquemia intestinal y formación de hernias; Charles A, et al. ${ }^{29}$ reportaron un caso de una mujer de 23 años de edad, quien en la semana 25 de embarazo presentó un cuadro clínico de isquemia intestinal en la anastomosis de la Y de Roux, diagnosticada por medio de endoscopia con un desenlace fatal, tanto para la madre como para el feto. Aunque la isquemia intestinal es una enfermedad infrecuente, es recomendable tener en mente esta patología en mujeres embarazadas que se han sometido a cirugía bariátrica al presentarse al servicio de urgencias con cuadros clínicos de dolor abdominal difuso.

Kakarla N, et al. reportaron 2 casos de hernias internas de Petersen en mujeres embarazadas que se 
sometieron a cirugía bariátrica. ${ }^{30}$ La hernia de Petersen es una complicación poco común, el espacio de Petersen se encuentra por debajo del asa de la Y de Roux que desciende para la anastomosis con el estómago. ${ }^{31}$ En Colombia sólo se ha reportado un caso de herniación por el defecto de Petersen, y fue en una mujer no embarazada que se sometió a un bypass gástrico. ${ }^{6}$

Deficiencias especificas de micronutrientes en la mujer embarazada son de preocupación después de la cirugía bariátrica; se han informado niveles bajos de ácido fólico y vitamina B12, después de una cirugía bariátrica y como consecuencia se puede producir un aumento de la homocisteína, ${ }^{32}$ aumentando el riesgo para las malformaciones del tubo neural, anemia megaloblástica, enfermedades neurológicas, enfermedades psiquiátricas, arteriosclerosis periférica, cerebral y coronaria, tromboembolismo recurrente, trombosis venosa profunda, infarto del miocardio y apoplejía isquémica. ${ }^{33,34}$

\section{Resultados maternos obstétricos y neonatales}

En cuanto a la relación entre la pérdida de peso materno y el peso neonatal al nacimiento, los estudios de años anteriores en los que aún se realizaba el puente yeyunoileal (cirugía bariátrica con componente malabsortivo) informaban estas complicaciones. ${ }^{35,36}$

Matielli, et al. en un estudio retrospectivo de serie de casos, reportó bajo peso al nacimiento como consecuencia de cirugía con componente de mala absorción o diversión gastrointestinal. ${ }^{37}$ En nuestra revisión encontramos sólo dos estudios prospectivos de serie de casos, que reportan bajo peso para la edad y/o nacimiento prematuro como complicación de procedimientos bariátricos. ${ }^{38,39}$

Sheiner, et al. estudiaron una población femenina entre 1988 y 2002, con el fin de hacer un seguimiento a los resultados del embarazo después de la cirugía bariátrica. De los 159.210 nacimientos durante el período de estudio, 298 fueron de pacientes que se habían sometido a procedimientos bariátricos; en el análisis multivariable no se observaron diferencias significativas en relación con el embarazo, características obstétricas y complicaciones (desprendimiento placentario, placenta previa, distocia, líquido amniótico teñido de meconio, mortalidad perinatal, malformaciones congénitas y bajos puntajes de APGAR al minuto y 5 minutos) entre los procedimientos abiertos $(n=159)$, los procedimientos laparoscópicos $(\mathrm{n}=139)$ y las mujeres que no se habían sometido a cirugías bariátricas. Sin embargo, encontraron mayores tasas de cesárea entre los grupos de cirugía bariátrica $(25,2 \%$ vs. 12,2\%, razón de momios, 2,4; 95\% intervalo de confianza, 1,9-3,1; $<<, 001$ ), con lo cual concluyeron que la cirugía bariátrica, si bien puede ser un factor de riesgo para cesárea, no se asoció con resultados perinatales adversos. ${ }^{40}$

Por otro lado, Dixon, et al. demuestran mediante un estudio prospectivo de serie de casos, utilizando análisis de regresión lineal en 79 mujeres, que no hay relación entre la pérdida de peso materno y el peso neonatal al nacimiento. ${ }^{41}$

Marceau, et al. mediante un estudio retrospectivo de serie de casos, aplicando un cuestionario de selección múltiple a 916 mujeres que fueron sometidas a cirugía bariátrica de tipo anastomosis biliopancreática, reportan que el $47 \%$ de las pacientes que no podían quedar en embarazo previo a la cirugía, tuvieron éxito luego de la misma. La incidencia de macrosomía fetal disminuyó de 34,8 a 7,7\%, con un aumento concomitante en los recién nacidos de peso normal de 62,1 a 82,7\%; los autores concluyen que la pérdida de peso después de la anastomosis biliopancreática mejora la función reproductiva de las mujeres con obesidad mórbida y así mismo proporciona importantes efectos benéficos para la madre y el niño, también recomiendan retrasar el embarazo en mujeres obesas hasta que la pérdida sustancial de peso, como consecuencia de la cirugía bariátrica, haya ocurrido. ${ }^{39}$ 
Bar-Zohar, et al. en un estudio prospectivo de serie de casos, siguieron a 74 mujeres operadas por medio de la banda gástrica, obteniendo una reducción del índice de masa corporal. Concluyen que el resultado del embarazo de mujeres obesas es peor, comparado con mujeres que tienen un índice de masa corporal normal; así mismo, la banda gástrica la consideran como un procedimiento seguro, tanto para la madre como para el hijo. ${ }^{42}$

Estudios más recientes, como el de Revaux A, et al. (retrospectivo de casos y controles), demuestran que la incidencia de resultados obstétricos adversos es mayor en las mujeres obesas que no se someten a banda gástrica (LABG: "Laparoscopic Adjustable Gastric Bandung”) que en las que se someten a este procedimiento en cuanto al aumento de la incidencia de preeclampsia, diabetes mellitus gestacional, bajo peso al nacer y macrosomía fetal. ${ }^{43}$

Las técnicas quirúrgicas, como la banda gástrica por laparoscopia (LABG), no predispone a deficiencias nutricionales si la ingesta es adecuada, ya que en este tipo de procedimientos no se producen resecciones ni anastomosis gastrointestinales, que en sí es el tipo de procedimiento que predispone a deficiencias nutricionales (hierro, vitamina B12 y calcio), en tales casos se recomienda un suplemento vitamínico adecuado. ${ }^{44,45}$ En general se sugiere un intervalo de 2 años entre la cirugía bariátrica. ${ }^{46}$ Por otra parte, se necesitan más estudios prospectivos diseñados cuidadosamente para confirmar estas observaciones y evaluar áreas de incertidumbre, como la resistencia a la insulina materna y neonatal, ${ }^{47}$ así como para evaluar la posibilidad de que la desnutrición relacionada con el desarrollo fetal sea precursora de algunas enfermedades de los adultos. ${ }^{48}$

\section{CONCLUSIÓN}

El tratamiento quirúrgico contra la obesidad surge como una alternativa, al parecer segura y efectiva. La evidencia muestra que los resultados del embarazo después de la cirugía bariátrica son favorables, en comparación con los resultados en los obesos no tratados quirúrgicamente.

\section{REFERENCIAS}

1. Haslam DW, James WP. Obesity. Lancet 2005;366:1197-1209.

2. Centers for Disease Control and Prevention (CDC). State-specific prevalence of obesity among adults -United States, 2005. MMWR Morb Mortal Wkly Rep 2006;55:985-8.

3. Ogden CL, Carroll MD, Curtin LR, McDowell MA, Tabak CJ, Flegal KM. Prevalence of overweight and obesity in the United States, 1999-2004. JAMA 2006;295:1549-55.

4. Wojciechowska E, Wilczyński W, Hincz P, Podciechowski L, Bielak A, Brot A, et al. Pregnancy and delivery after bariatric surgery. Ginekol Pol 2006;77:926-9.

5. Profamilia. Encuesta Nacional de demografía y salud 2005. Salud Sexual y Reproductiva en Colombia; 2005. p. 274-75.

6. Cañadas R, Lombana LJ, Hernández J, Solano C, Suárez J, Torres D, et al. Complicaciones de cirugía bariátrica. Hernia interna: una condición potencialmente fatal. Reporte de un caso y revisión de la literatura. Rev Col Gastroenterol 2007;22:243-8.

7. Maggard MA, Shugarman LR, Suttorp M, Maglione M, Sugarman HJ, Livingston EH, et al. Meta-analysis: surgical treatment of obesity. Ann Intern Med 2005;142:547-59.

8. Li Z, Maglione M, Tu W, Mojica W, Arterburn D, Shugarman LR, et al. Meta-analysis: pharmacologic treatment of obesity. Ann Intern Med 2005;142:532-46.

9. Buchwald H, Williams SE. Bariatric surgery worldwide 2003. Obes Surg 2004;14:1157-64.

10. Chapman A, Game P, O’Brien P, Maddern G, Kiroff G, Foster B, Ham J. Executive summary: Laparoscopic adjustable gastric banding for the treatment of obesity: Update and re-appraisal. Australian Safety and Efficacy Register of New Interventional Procedures-Surgical (ASERNIP-S) Report No. 31. Second Edition. Adelaide, South Australia: ASERNIP-S; June 2002.

11. DeMaria EJ, Jamal MK. Laparoscopic adjustable gastric banding: evolving clinical experience. Surg Clin North Am 2005;85:773-87.

12. Li Z, Bowerman S, Heber D. Health ramifications of the obesity epidemic. Surg Clin North Am 2005;85:681-701.

13. Cnattingius S, Bergström R, Lipworth L, Kramer MS. Repregnancy weight and the risk of adverse pregnancy outcomes. N Engl J Med 1998;338:147-52. 
14. Cedergren MI. Maternal morbid obesity and the risk of adverse pregnancy outcome. Obstet Gynecol 2004;103:219-24.

15. Morin KH, Reilly L. Caring for obese pregnant women. J Obstet Gynecol Neonatal Nurs 2007;36:482-9.

16. American College of Obstetricians and Gynecologists. ACOG Committee Opinion number 315, September 2005. Obesity in pregnancy Obstet Gynecol 2005;106:671-5.

17. Perlow JH, Morgan MA, Montgomery D, Towers CV, Porto M. Perinatal outcome of pregnancy complicated by massive obesity. Am J Obstet Gynecol 1992;167:958-62.

18. Institute of Medicine. Subcommittee on Nutritional Status and Weight Gain during Pregnancy. Nutrition during pregnancy. I. Weight gain. II. Nutritional supplements. Washington, DC: National Academy Press; 1990. p. 1-13.

19. Deitel M, Stone E, Kassam H, Wilk E, Sutherland D. Gynecologic-obstetric changes after loss of massive excess weight following bariatric surgery. J Am Coll Nutr 1988;7:147-53.

20. Wittgrove AC, Jester L, Wittgrove P, Clark GW. Pregnancy following gastric bypass for morbid obesity. Obes Surg 1998;8:461-4.

21. Dixon JB, Dixon ME, O'Brien PE. Pregnancy after Lap-Band surgery: management of the band to achieve healthy weight outcomes. Obes Surg 2001;11:59-65.

22. Roehrig HR, Xanthakos SA, Sweeney J, Zeller MH, Inge TH. Pregnancy after gastric bypass surgery in adolescents. Obes Surg 2007;17:873-7.

23. Patel JA, Colella JJ, Esaka E, Patel NA, Thomas RL. Improvement in infertility and pregnancy outcomes after weight loss surgery. Med Clin North Am 2007;91:515-28, xiii.

24. Sugerman HJ, Starkey JV, Birkenhauer R. A randomized prospective trial of gastric bypass versus vertical banded gastroplasty for morbid obesity and their effects on sweets versus non-sweets eaters. Ann Surg 1987;205:613-24.

25. McMahon MM, Sarr MG, Clark MM, Gall MM, Knoetgen J 3rd, Service FJ, et al. Clinical management after bariatric surgery: value of a multidisciplinary approach. Mayo Clin Proc 2006;81:S34-S45.

26. Kaplan LM. Gastrointestinal management of the bariatric surgery patient. Gastroenterol Clin North Am 2005;34:105-25.
27. Nguyen NT, Silver M, Robinson M, Needleman B, Hartley G, Cooney R, et al. Result of a national audit of bariatric surgery performed at academic centers: a 2004 University Health System Consortium benchmarking project. Arch Surg 2006;141:445-50.

28. Zingmond DS, McGory ML, Ko CY. Hospitalization before and after gastric bypass surgery. JAMA 2005;294:1918-24.

29. Charles A, Domingo S, Goldfadden A, Fader J, Lampmann R, Mazzeo R. Small bowel ischemia after Roux-en-Y gastric bypass complicated by pregnancy: a case report. Am Surg 2005;71:231-4.

30. Kakarla N, Dailey C, Marino T, Shikora SA, Chelmow D. Pregnancy after gastric bypass surgery and internal hernia formation. Obstet Gynecol. 2005;105:1195-8.

31. Higaa K, Boonea K, Arteaga González I, LópezTomassetti Fernández E. Mesenteric closure in laparoscopic gastric bypass: surgical technique and literature review. Cir Esp 2007;82:77-88.

32. Dixon JB, Dixon ME, O'Brien PE. Elevated homocysteine levels with weight loss after Lap-Band surgery: higher folate and vitamin B12 levels required to maintain homocysteine level. Int J Obes Relat Metab Disord 2001;25:219-27.

33. Booth GL, Wang EE. Preventive health care, 2000 update: screening and management of hyperhomocysteinemia for the prevention of coronary artery disease events. The Canadian Task Force on Preventive Health Care. CMAJ 2000;163:21-9.

34. Toole JF, Malinow MR, Chambless LE, Spence JD, Pettigrew LC, Howard VJ, et al. Lowering homocysteine in patients with ischemic stroke to prevent recurrent stroke, myocardial infarction, and death: the Vitamin Intervention for Stroke Prevention (VISP) randomized controlled trial. JAMA 2004;291:565-75.

35. Knudsen LB, Källén B. Intestinal bypass operation and pregnancy outcome. Acta Obstet Gynecol Scand 1986;65:831-4.

36. Ingardia CJ, Fischer JR. Pregnancy after jejunoileal bypass and the SGA infant. Obstet Gynecol 1978;52:215-8.

37. Matielli J, Garrido AB Jr, Oliveira MR, Berti LV, Elias AA. Pregnancy following silicone ring gastric bypass. Obes Surg 2004;14:916.

38. Marceau P, Kaufman D, Biron S, Hould FS, Lebel S, Marceau S, et al. Outcome of pregnancies after biliopancreatic diversion. Obes Surg 2004;14:318-24. 
39. Friedman D, Cuneo S, Valenzano M, Marinari GM, Adami GF, Gianetta E, et al. Pregnancies in an 18-year follow-up after biliopancreatic diversion. Obes Surg 1995;5:308-13.

40. Sheiner E, Levy A, Silverberg D, Menes TS, Levy I, Katz M, et al. Pregnancy after bariatric surgery is not associated with adverse perinatal outcome. Am J Obstet Gynecol 2004;190:1335-40.

41. Dixon JB, Dixon ME, O'Brien PE. Birth outcomes in obese women after laparoscopic adjustable gastric banding. Obstet Gynecol 2005;106:965-72.

42. Bar-Zohar D, Azem F, Klausner J, Abu-Abeid S. Pregnancy after laparoscopic adjustable gastric banding: perinatal outcome is favourable also for women with relatively high gestational weight gain. Surg Endosc 2006;20:1580-3.

43. Ducarme G, Revaux A, Rodriguez A, Aissaoui F, Pharisien I, Uzan M. Obstetric outcome following laparoscopic adjustable gastric banding. Int J Gynaecol Obstet 2007;98:244-7.
44. Brolin RE, Gorman RC, Milgrim LM, Kenler HA. Multivitamin prophylaxis in prevention of post-gastric bypass vitamin and mineral deficiencies. Int J Obes 1991;15:661-7.

45. Brolin RE, Gorman JH, Gorman RC, Petschenik AJ, Bradley LB, Kenler HA, et al. Prophylactic iron supplementation after Roux-en-Y gastric bypass: a prospective, double-blind, randomized study. Arch Surg 1998;133:740-4.

46. Dao T, Kuhn J, Ehmer D, Fisher T, McCarty T. Pregnancy outcomes after gastric-bypass surgery. Am J Surg 2006;192:762-6.

47. Kral JG, Brolin RE, Buchwald H, Pories WJ, Sarr MG, Sugerman HJ, et al. Research considerations in obesity surgery. American Society for Bariatric Surgery, Gainesville, Florida, USA. Obes Res 2002;10:63-4.

48. Prentice AM. Intrauterine factors, adiposity, and hyperinsulinaemia. BMJ 2003;327:880-1. 